OU-HET/179

hep-th/9305170

May 1993

\title{
Transition Amplitude in $2+1$ dimensional Chern-Simons Gravity on a Torus
}

\author{
Kiyoshi Ezawa円 \\ Department of Physics \\ Osaka University, Toyonaka, Osaka 560, Japan
}

\begin{abstract}
In the framework of the Chern-Simons gravity proposed by Witten, a transition amplitude of a torus universe in $2+1$ dimensional quantum gravity is computed. This amplitude has the desired properties as a probability amplitude of the quantum mechanics of a torus universe, namely, it has a peak on the "classical orbit" and it satisfies the Shrödinger equation of the $2+1$ dimentional gravity. The discussion is given that the classical orbit dominance of the amplitude is not altered by taking the modular invariance into account and that this amplitude can serve as a covariant transition amplitude in a particular sense. A set of the modular covariant wavefunctions is also constructed and they are shown to be equivalent with the weight-1/2 Maass forms.
\end{abstract}

\footnotetext{
${ }^{1}$ e-mail address: ezawa@oskth.kek.jp
} 


\section{Introduction}

For more than ten years, attention has been paid to the $2+1$ dimensional gravity [1] [2] as a useful toy model which gives insights into the $3+1$ dimensional quantum gravity. In $2+1$ dimensions, the vacuum Einstein equation with vanishing cosmological constant requires the spacetime manifolds to be locally flat. The $2+1$ dimensional Einstein gravity is therefore described by a finite number of global degrees of freedom [1]]. It has been shown by Witten that this $2+1$ dimensional Einstein gravity is equivalent to the $\operatorname{ISO}(2,1)$ gauge theory represented by the Chern-Simons action [3]. Many people have studied this

"Chern-Simons gravity" [4 since then. Some of them investigated relations between the C-S gravity and the $2+1$ dimensional ADM formalism [5] [6].

Most of these results are, however, formal in the sense that it is hard to extract physical pictures from them. There are some works which deal with physical processes by topological ideas on path integrals in the WKB approximation [7]. There appears to be no work which determines the quantum evolution of moduli in a fixed spatial topology.

In this paper, we compute a transition amplitude in the $2+1$ dimensional quantum gravity that describes the evolution of moduli of the space with a definite topology ( here, we investigate the most tractable topology, i.e. a torus $T^{2}$ ). Our strategy is the following. The ADM formalism [8] [9] is suitable for following the evolution of space manifolds. The complicated Hamiltonian, however, makes its quantization very hard. While the C-S gravity can be quantized in a simple way, it is difficult to give physical interpretations. We exploit both formalisms. By using relations between these two formalisms studied by Carlip [5] and by solving the Schrödinger equation of the quantum C-S gravity, we compute the transition amplitude describing a time-evolution of the moduli parameters, which are basic configuration variables of the ADM formalism.

This transition amplitude has a divergent peak on the classical orbit. It satisfies the Schrödinger equation of the ADM formalism, and possesses required properties as a probability amplitude of the $2+1$ dimensional quantum gravity. In particular it turns out that our amplitude serves as a modular covariant transition amplitude when the integration region of the inner product is extended to the upper-half plane.

Besides, a set of modular covariant wavefunctions, which are the eigenfunctions of the volume operator, are constructed. It is shown that these wavefunctions are related with 
the weight-1/2 Maass forms [14] by way of the integral transformation.

In $\S 2$ we solve the Hamilton equations in the ADM formalism to investigate the classical evolution of a torus universe. There, the classical orbit is also found out. $\S 3$ is devoted to the review of the classical and quantum relations between the ADM formalism and the C-S gravity studied by Carlip. From these results in $\S 3$, the transition amplitude of a torus universe is computed in $\S 4$. Its properties are examined and we show that it can be interpreted as a probability amplitude. The main result in $\$ 5$ is the equivalence of eq.(55) with eq.(54), owing to which our amplitude can be regarded to be modular covariant in a sense. We will also show that physics is not modified after taking the modular invariance into account. The set of covariant wavefunctions is also given in this section. In $\S 6$, after summarizing our results, we discuss a possibility to generalize our method to more general contexts.

\section{The classical orbit of a torus universe}

The 2+1 dimensional ADM formalism on a torus is formulated by Moncrief [8] and Hosoya and Nakao [9] [10]. In this section, we briefly review this ADM formalism on a torus, and look into the classical behavior of the torus universe by solving the Hamilton equations given by the reduced ADM action.

In the $2+1$ dimensional ADM formalism, the spacetime manifold $M$ is assumed to be homeomorphic to $R^{1} \times \Sigma$, where $\Sigma$ is a two dimensional space called time-slice. Then, the ADM action is given by

$$
I_{A D M}=\int d t \int_{\Sigma} d^{2} x\left(\pi^{a b} \dot{g}_{a b}-N^{a} \mathcal{H}_{a}-N \mathcal{H}\right)
$$

where $g_{a b}$ is the induced metric on $\Sigma, \pi^{a b}$ is its conjugate momentum, $N^{a}$ is the shift vector, $\mathrm{N}$ is the lapse function, and $\mathcal{H}_{a}$ and $\mathcal{H}$ are respectively the momentum and Hamiltonian constraint. Here, we further assume that the time-slice $\Sigma$ has the topology of a torus $T^{2}$. On taking York's time-slice, we find that eq.(1) reduces to

$$
I_{A D M}^{*}=\int d t\left(p_{1} \dot{m}_{1}+p_{2} \dot{m}_{2}+\tau \dot{v}-N^{\prime} \mathcal{H}^{\prime}\right)
$$

where $m_{A}$ and $p_{A}(\mathrm{~A}=1,2)$ are the moduli parameters and their conjugate momenta respectively, $N^{\prime} \equiv N / 2 v$, and

$$
\mathcal{H}^{\prime}=m_{2}^{2}\left\{\left(p_{1}\right)^{2}+\left(p_{2}\right)^{2}\right\}-v^{2} \tau^{2} .
$$


With York's time-slice, the mean curvature $\tau \equiv \sqrt{g}^{-1} g_{a b} \pi^{a b}$ is constant everywhere on $\Sigma$. The conformal factor ( the "volume" ) $v \equiv \sqrt{\operatorname{det}\left(g_{a b}\right)}$ is a global scalar quantity independent of spatial coordinates.

This reduced ADM action (2) has a first class constraint $\mathcal{H}^{\prime} \approx 0$. We may use this reduced action to see the classical behavior of the torus universe. Alternatively, we use the gauge-fixed system. We impose a coordinate condition

$$
\tau=t(=\text { time coordinate })
$$

and solve the constraint $\mathcal{H}^{\prime} \approx 0$ explicitly. We find

$$
I_{A D M}^{* *}=\int d \tau\left(p_{1} \dot{m}_{1}+p_{2} \dot{m}_{2}-H\right),
$$

where the dot denotes a derivative with respect to $\tau$, and the Hamiltonian $H$ of this system is

$$
H \equiv v=\frac{m_{2}}{\tau} \sqrt{\left(p_{1}\right)^{2}+\left(p_{2}\right)^{2}} .
$$

Let us use eqs.(5) and (6) to investigate the time evolution of the torus universe.

First, we solve the Hamilton equations:

$$
\frac{d}{d \tau}\left(m_{A}, p_{A}\right)=\left\{\left(m_{A}, p_{A}\right), H\right\}_{P . B .},(A=1,2) .
$$

The results are

$$
\begin{aligned}
& m_{1}=\frac{\frac{L+E}{\tau_{0}}+\frac{\tau_{0}(L-E)}{\tau^{2}}}{\frac{p_{1}}{\tau_{0}}+\frac{p_{1} \tau_{0}}{\tau^{2}}} \\
& m_{2}=\frac{p_{1}}{\left|p_{1}\right|} \frac{2 \frac{E}{\tau}}{\frac{p_{1}}{\tau_{0}}+\frac{p_{1} \tau_{0}}{\tau^{2}}} \\
& p_{1}=\text { constant with respect to } \tau \\
& p_{2}=-\frac{\tau}{2}\left(\frac{\left|p_{1}\right|}{\tau_{0}}-\frac{\left|p_{1}\right| \tau_{0}}{\tau^{2}}\right),
\end{aligned}
$$

where $E, L, \tau_{0}$, and $p_{1}$ are the constants of motion.

We will explicitly construct a spacetime metric in $2+1$ dimensions from this solution. Let us determine the lapse function and the shift vector. Following Moncrief [ [8], the lapse $N$ is found to be obtained from the Hamilton equation in the constrained system $1 \equiv \frac{\partial \tau}{\partial t}=N^{\prime}\left\{\tau, \mathcal{H}^{\prime}\right\}_{P . B .}=N \tau^{2}$

$$
N=\frac{1}{\tau^{2}} \text {. }
$$


The shift $N^{a}$ can be absorbed by a redefinition of the origin of each time-slice. Henceforth, we put

$$
N^{a}=0
$$

From eqs.(8)-(10), we can compute the spacetime metric as

$$
\begin{aligned}
d s^{2}= & -(N d \tau)^{2}+\frac{v}{m_{2}}\left\{\left(d x+m_{1} d y\right)^{2}+\left(m_{2} d y\right)^{2}\right\} \\
= & -\frac{d \tau^{2}}{\tau^{4}}+\frac{1}{\tau^{2}}\left(\sqrt{\frac{\left|p_{1}\right| \tau_{0}}{2}} d x+\sqrt{\frac{\left|p_{1}\right| \tau_{0}}{2}} \frac{L-E}{p_{1}} d y\right)^{2} \\
& +\left(\sqrt{\frac{\left|p_{1}\right|}{2 \tau_{0}}} d x+\sqrt{\frac{\left|p_{1}\right|}{2 \tau_{0}}} \frac{L+E}{p_{1}} d y\right)^{2},
\end{aligned}
$$

where $(x, y)$ is the coordinate on time-slice $\Sigma$ with the periodic condition

$$
(x, y) \sim(x+1, y) \sim(x, y+1) .
$$

The spacetime equipped with this metric turns out to be embedded into the Minkowski space. To see this explicitly, we transform the coordinates in two steps. First,

$$
\left\{\begin{array}{l}
\tau=\tau \\
\theta=\sqrt{\frac{\left|p_{1}\right| \tau_{0}}{2}} x+\sqrt{\frac{\left|p_{1}\right| \tau_{0}}{2}} \frac{L-E}{\frac{p_{1}}{p_{1}}} y \\
Y=-\frac{p_{1}}{\left|p_{1}\right|}\left(\sqrt{\frac{\left|p_{1}\right|}{2 \tau_{0}}} x+\sqrt{\frac{\left|p_{1}\right|}{2 \tau_{0}}} \frac{L+E}{p_{1}} y\right) .
\end{array}\right.
$$

Second,

$$
\left\{\begin{array}{l}
T=\frac{1}{\tau} \cosh \theta \\
X=\frac{1}{\tau} \sinh \theta \\
Y=Y
\end{array}\right.
$$

The metric (11) becomes

$$
d s^{2}=-\frac{d \tau^{2}}{\tau^{4}}+\frac{1}{\tau^{2}} d \theta^{2}+d Y^{2}=-d T^{2}+d X^{2}+d Y^{2}
$$

Thus, the torus universe $M$ is regarded as a quotient space

$$
M=\mathcal{F} / G
$$

We denote by $\mathcal{F}=\{(T, X, Y)|T>| X \mid\}$ the "fundamental region" in the Minkowski space, and by $G$ a discrete subgroup of $\operatorname{ISO}(2,1)$ generated by the following two transformations.

$$
\begin{aligned}
& \Lambda_{1}:(T, X, Y) \rightarrow(T \cosh a+X \sinh a, T \sinh a+X \cosh a, Y+u) \\
& \Lambda_{2}:(T, X, Y) \rightarrow(T \cosh b+X \sinh b, T \sinh b+X \cosh b, Y+w),
\end{aligned}
$$


where

$$
\begin{aligned}
& (a, u) \equiv\left(\sqrt{\frac{\tau_{0}\left|p_{1}\right|}{2}},-\frac{p_{1}}{\left|p_{1}\right|} \sqrt{\frac{\left|p_{1}\right|}{2 \tau_{0}}}\right) \\
& (b, w) \equiv\left(\frac{L-E}{p_{1}} \sqrt{\frac{\tau_{0}\left|p_{1}\right|}{2}},-\frac{L+E}{\left|p_{1}\right|} \sqrt{\frac{\left|p_{1}\right|}{2 \tau_{0}}}\right) .
\end{aligned}
$$

Therefore, the evolution of the torus universe can be visualized as illustrated in Fig.1, and is determined by the four time-independent parameters $(a, b, u, w)$.

Next, we determine the classical orbit. Here we mean by "classical orbit" a curve drawn by the point $\left(m_{1}^{\prime}, m_{2}^{\prime}\right)$ in the moduli space, which the torus reaches at time $\tau_{2}$ by way of classical trajectories, assuming that the torus left the point $\left(m_{1}, m_{2}\right)$ at time $\tau_{1}$. It will be useful in $\S 4$ when we discuss the properties of the transition amplitude in the quantum theory. To this end, we first express the moduli parameters in terms of the parameters $(a, b, u, w)$ using eqs. (8), (18).

$$
m_{1}(\tau)=\frac{u w+\frac{a b}{\tau^{2}}}{u^{2}+\frac{a^{2}}{\tau^{2}}}, m_{2}(\tau)=\frac{\frac{-a w+b u}{\tau}}{u^{2}+\frac{a^{2}}{\tau^{2}}},
$$

where we have shown explicitly that the moduli parameters are the $\tau$-dependent functions.

In general, we have four free parameters $(a, b, u, w)$ in (19). Two of them are fixed when we impose an initial condition

$$
m_{1}\left(\tau_{1}\right)=m_{1}, m_{2}\left(\tau_{1}\right)=m_{2}
$$

We solve this set of equations for parameters $(b, w)$ and find

$$
b=a m_{1}+\tau_{1} u m_{2}, w=u m_{1}-\frac{a}{\tau_{1}} m_{2} .
$$

Substituting eq.(21) into

$$
m_{1}\left(\tau_{2}\right)=m_{1}^{\prime} \text { and } m_{2}\left(\tau_{2}\right)=m_{2}^{\prime}
$$

we finally obtain

$$
\begin{aligned}
& m_{1}^{\prime}=m_{1}+\frac{m_{2}}{2}\left(\frac{\tau_{1}}{\tau_{2}}-\frac{\tau_{2}}{\tau_{1}}\right) \frac{2 x}{1+x^{2}} \\
& m_{2}^{\prime}=\frac{m_{2}}{2}\left(\frac{\tau_{2}}{\tau_{1}}+\frac{\tau_{1}}{\tau_{2}}\right)+\frac{m_{2}}{2}\left(\frac{\tau_{1}}{\tau_{2}}-\frac{\tau_{2}}{\tau_{1}}\right) \frac{1-x^{2}}{1+x^{2}},
\end{aligned}
$$


where $x \equiv a /\left(u \tau_{2}\right)$. It is easy to see that, when $x$ varies in the range $(-\infty,+\infty)$, the point $\left(m_{1}^{\prime}, m_{2}^{\prime}\right)$ in the moduli space moves on the curve:

$$
C_{\text {c.o. }}:\left(m_{1}^{\prime}-m_{1}\right)^{2}+\left\{m_{2}^{\prime}-\frac{m_{2}}{2}\left(\frac{\tau_{2}}{\tau_{1}}+\frac{\tau_{1}}{\tau_{2}}\right)\right\}^{2}=\left\{\frac{m_{2}}{2}\left(\frac{\tau_{2}}{\tau_{1}}-\frac{\tau_{1}}{\tau_{2}}\right)\right\}^{2} .
$$

As is illustrated in Fig.2, this classical orbit is a circle in the moduli spacef, expanding with time $\tau_{2}$. In the limit $\tau_{2} \rightarrow \infty$ (or 0 ), the circle approaches the $m_{1}^{\prime}$-axis plus infinity.

\section{The relations between Chern-Simons gravity and ADM formalism}

The classical and the quantum equivalence of the C-S gravity to the ADM formalism is investigated by Carlip [5]. In this section, we review his work, putting an emphasis on the quantum relation.

According to Witten [3], the physical phase space of the C-S gravity is the moduli space $\mathcal{M}$ of flat $\operatorname{ISO}(2,1)$ connections on $\Sigma$. When $\Sigma$ has the topology of a torus $T^{2}$, the moduli space $\mathcal{M}$ is parametrized by two commuting elements of $\operatorname{ISO}(2,1)$ up to conjugation. This phase space is known to have three disconnected sectors [11] which are denoted by

the timelike sector $\mathcal{M}_{t}$, the null sector $\mathcal{M}_{n}$ and the spacelike sector $\mathcal{M}_{s}$.

These three sectors are characterized by the restriction of their $\operatorname{ISO}(2,1)$ transformations to $\mathrm{SO}(2,1)$, which are two spatial rotations, null rotations and Lorentz boosts respectively. Here we pick the spacelike sector $\mathcal{M}_{s}$ which is physically most relevant. Taking a proper conjugation, two $\operatorname{ISO}(2,1)$ transformations which coordinatize the phase space can be expressed as follows;

$$
\begin{aligned}
& \Lambda_{1}:(T, X, Y) \rightarrow(T \cosh a+X \sinh a, T \sinh a+X \cosh a, Y+u) \\
& \Lambda_{2}:(T, X, Y) \rightarrow(T \cosh b+X \sinh b, T \sinh b+X \cosh b, Y+w) .
\end{aligned}
$$

This is precisely the same expression as eq.(17). Thus in the C-S gravity, given a point in $\mathcal{M}_{s}$, a spacetime manifold $M$ can be constructed as in eq.(16). Moreover, a detailed analysis shows the free parameters $(a, b ; w, u)$ to be canonical coordinates of the C-S

\footnotetext{
${ }^{2}$ More precisely, this circle is in the upper-half plane $\left\{\left(m_{1}^{\prime}, m_{2}^{\prime}\right) \mid m_{2}^{\prime}>0\right\}$. The true moduli space is a quotient space of this upper-half plane modulo the action of the modular group $\Gamma$.
} 
gravity on a torus [5]. With these facts, we can write the basic variables $\left(m_{1}, m_{2} ; p_{1}, p_{2}\right)$ in the $\mathrm{ADM}$ in terms of $(a, b ; w, u)$. The moduli parameters are given in eq.(19) and the conjugate momenta are

$$
p_{1}=-2 a u, p_{2}=-\tau\left(u^{2}-\frac{a^{2}}{\tau^{2}}\right)
$$

The ADM Hamiltonian eq.(6) is written as

$$
H=\frac{-a w+b u}{\tau} .
$$

Using eqs.(19) and (25), we can relate the C-S gravity to the ADM formalism by a time-dependent canonical transformation

$$
p_{1} d m_{1}+p_{2} d m_{2}-H d \tau=-2 u d b+2 w d a+d F
$$

where

$$
F\left(m_{1}, m_{2}, a, b ; \tau\right)=\frac{1}{m_{2} \tau}\left[\left(b-m_{1} a\right)^{2}+\left(m_{2} a\right)^{2}\right]
$$

is the generating function. From this relation, we see the Hamiltonian $H_{C-S}$ which generates the time-evolution in the C-S gravity vanishes,

$$
H_{C-S} \equiv H+\frac{\partial}{\partial \tau} F=0
$$

Here we should note that while a point in the phase space of the C-S gravity is stable under the $\tau$-evolution, it determines the evolution of the torus universe with $\tau$ as in Fig. 1.

Let us now see the quantum relation. We construct operators which represent the ADM-variables in the Hilbert space of the C-S gravity. The fundamental operators in the C-S gravity are the self-adjoint ones $\hat{a}, \hat{b}, \hat{w}$ and $\hat{u}$ corresponding to the canonical variables, whose canonical commutation relations are

$$
[\hat{a}, \hat{w}]=-[\hat{b}, \hat{u}]=\frac{i}{2}
$$

We can construct the basic operators in the ADM by replacing eqs.(19) and (25) by the corresponding operator relations

$$
\begin{aligned}
& \hat{m} \equiv \hat{m}_{1}+i \hat{m}_{2}=\left[\hat{u}+i \frac{\hat{a}}{\tau}\right]^{-1}\left[\hat{w}+i \frac{\hat{b}}{\tau}\right], \\
& \hat{p} \equiv \hat{p}_{1}+i \hat{p}_{2}=-i \tau\left[\hat{u}-i \frac{\hat{a}}{\tau}\right]^{2} .
\end{aligned}
$$


The ADM Hamiltonian is expressed as

$$
\hat{H}=\frac{-\hat{a} \hat{w}+\hat{u} \hat{b}}{\tau} .
$$

Here, we adopt the $(\hat{a}, \hat{b})$-diagonalized representation, where the wave function $\chi$ is a function of $a$ and $b$. We adopt a natural inner product proposed by Ashtekar et.al. [11]

$$
<\chi_{1} \mid \chi_{2}>=\iint d a d b \overline{\chi_{1}(a, b)} \chi_{2}(a, b),
$$

where the bar denotes complex conjugate. The fundamental operators act on the wave function as

$$
\begin{aligned}
& \hat{a} \chi=a \cdot \chi, \hat{b} \chi=b \cdot \chi \\
& \hat{w} \chi=-\frac{i}{2} \frac{\partial}{\partial a} \chi, \hat{u} \chi=\frac{i}{2} \frac{\partial}{\partial b} \chi
\end{aligned}
$$

In this representation, the eigenfunction of the moduli operators $\left(\hat{m}, \hat{m}^{\dagger}\right)$ eq.(30) is known to be

$$
K(m, \bar{m} ; a, b, \tau)=\frac{b-m a}{\pi \tau \sqrt{2 m_{2}}} \exp \left(-\frac{i}{m_{2} \tau}|b-m a|^{2}\right)
$$

which satisfies

$$
\hat{m} K=m \cdot K \text { and } \hat{m}^{\dagger} K=\bar{m} \cdot K
$$

This $K$ possesses the following properties;

$$
\begin{gathered}
\text { orthogonality : } \quad \iint d a d b \overline{K\left(m^{\prime}, \bar{m}^{\prime} ; a, b, \tau\right)} K(m, \bar{m} ; a, b, \tau) \\
=m_{2}^{2} \delta^{2}\left(m-m^{\prime}\right)
\end{gathered}
$$

time dependence : $\quad-i \frac{\partial}{\partial \tau} K(m, \bar{m} ; a, b, \tau)=\hat{H} K(m, \bar{m} ; a, b, \tau)$.

Using this $K$ as a kernel, we can define the integral transformation from the $\left(\hat{m}, \hat{m}^{\dagger}\right)$ diagonal representation $\tilde{\chi}(m, \bar{m})$ to the $(\hat{a}, \hat{b})$-diagonal one $\chi(a, b)$

$$
\chi(a, b)=\iint \frac{d^{2} m}{m_{2}^{2}} K(m, \bar{m} ; a, b, \tau) \tilde{\chi}(m, \bar{m}),
$$

where the domain of integration is taken to be the upper-half complex $m$-plane. Inserting eq. (39) into eq. (33) and using the orthogonality (37), we obtain the modular-invariant inner-product in the $\left(\hat{m}, \hat{m}^{\dagger}\right)$-diagonal representation

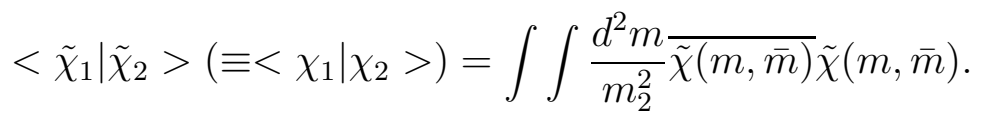


We can set up a quantization of the ADM formalism using $\tilde{\chi}$ which transforms as the "spinor representation" under the modular transformation [5]. In particular, the ADM Hamiltonian operator takes the form,

$$
\hat{H}=\frac{1}{\tau}\left[m_{2}\left(\hat{p}^{\dagger} \hat{p}\right)^{1 / 2}-\frac{1}{2}\left(\hat{p}^{\dagger} \hat{p}\right)^{-1 / 2} \hat{p}\right]=\tau^{-1}\left(\Delta_{1 / 2}\right)^{1 / 2},
$$

where

$$
\Delta_{1 / 2} \equiv-m_{2}^{2}\left(\frac{\partial^{2}}{\partial m_{1}^{2}}+\frac{\partial^{2}}{\partial m_{2}^{2}}\right)+i m_{2} \frac{\partial}{\partial m_{1}}-\frac{1}{4}
$$

is the Maass Laplacian [12] for automorphic forms of weight $\frac{1}{2}$. With this Hamiltonian, the time evolution of the wave function $\tilde{\chi}$ is described by the Schrödinger equation

$$
i \frac{\partial}{\partial \tau} \tilde{\chi}(m, \bar{m} ; \tau)=\tau^{-1}\left(\Delta_{1 / 2}\right)^{1 / 2} \tilde{\chi}(m, \bar{m} ; \tau) .
$$

On the other hand, the following Schrödinger equation holds in the C-S gravity:

$$
i \frac{\partial}{\partial \tau} \chi(a, b)=0,
$$

since we have vanishing Hamiltonian. Eq.(38) is necessary for $\tilde{\chi}$ and $\chi$ which are related via eq.(39) to satisfy eqs. (42) and (43) respectively. Thus, we can, at least formally, show that for a solution $\chi(a, b)$ of eq. (43), its inverse transform

$$
\tilde{\chi}(m, \bar{m})=\iint d a d b \overline{K(m, \bar{m} ; a, b, \tau)} \chi(a, b)
$$

solves the Schrödinger equation (42).

\section{The transition amplitude of a torus universe}

In the last section, we have given a framework of the quantum C-S gravity on a torus. Let us now calculate the transition amplitude of the torus universe.

Equation (43) tells us that the Schrödinger wave function in the C-S gravity is a $\tau$-independent function of configuration variables $a, b$. Therefore, the eigenfunction of moduli parameters $K(m, \bar{m} ; a, b, \tau)$ is not a solution to eq. (43). However, the wave function

$$
K\left(m, \bar{m} ; a, b, \tau_{1}\right)=\frac{b-m a}{\pi \tau_{1} \sqrt{2 m_{2}}} \exp \left(-\frac{i}{m_{2} \tau_{1}}|b-m a|^{2}\right)
$$


obtained by replacing $\tau$ with a (positive) constant $\tau_{1}$, has no time dependence and becomes a Schrödinger wave function. It is interpreted as a state under which the moduli parameters $\hat{m}, \hat{m}^{\dagger}$ have an eigenvalue $m, \bar{m}$ at the moment $\tau=\tau_{1}$, and keeps its form during the evolution. Similarly, the wave function $K\left(m^{\prime}, \bar{m}^{\prime} ; a, b, \tau_{2}\right)$ is the state for which the eigenvalues of the moduli parameters at $\tau=\tau_{2}$ are $m^{\prime}$ and $\overline{m^{\prime}}$.

If we regard the inner product (33) to be a probability amplitude as in the standard quantum mechanics, the transition amplitude from moduli $m$ at $\tau=\tau_{1}$ to moduli $m^{\prime}$ at $\tau=\tau_{2}$ is written as

$$
\begin{aligned}
& <m, \overline{m^{\prime}} ; \tau_{2} \mid m, \bar{m} ; \tau_{1}>=\iint d a d b \overline{K\left(m^{\prime}, \bar{m}^{\prime} ; a, b, \tau_{2}\right)} K\left(m, \bar{m} ; a, b, \tau_{1}\right) \\
& =\int_{-\infty}^{\infty} d a \int_{-\infty}^{\infty} d b \frac{\left(b-\bar{m}^{\prime} a\right)(b-m a)}{2 \pi^{2} \tau_{1} \tau_{2} \sqrt{m_{2} m_{2}^{\prime}}} \exp \left\{\frac{i}{m_{2}^{\prime} \tau_{2}}\left|b-m^{\prime} a\right|^{2}-\frac{i}{m_{2} \tau_{1}}|b-m a|^{2}\right\}
\end{aligned}
$$

This expression involves the integral of the Fresnel-type $\int d x x^{2} \exp \left(i \alpha x^{2}\right)$, and we regularize it by the $i \epsilon$-prescription:

$$
\int_{-\infty}^{\infty} d x x^{2} e^{i \alpha x^{2}}=\frac{i}{2 \alpha} \sqrt{\frac{i \pi}{\alpha+i \epsilon}}, \epsilon>0 .
$$

With this regularization, eq.46) becomes

$$
\begin{aligned}
& <m^{\prime}, \overline{m^{\prime}} ; \tau_{2} \mid m, \bar{m} ; \tau_{1}> \\
& =\frac{1}{4 \pi} \frac{m_{2} m_{2}^{\prime}}{\sqrt{\tau_{1} \tau_{2}}} \frac{\left(\tau_{1}-\tau_{2}\right)\left(\bar{m}^{\prime}-m\right)}{\left\{m_{2}^{\prime 2}+m_{2}^{2}-m_{2} m_{2}^{\prime}\left(\frac{\tau_{1}}{\tau_{2}}+\frac{\tau_{2}}{\tau_{1}}\right)+\left(m_{1}^{\prime}-m_{2}\right)^{2} \mp i \epsilon\right\}^{3 / 2}},
\end{aligned}
$$

where $-i \epsilon(+i \epsilon)$ corresponds to the case of $m_{2} \tau_{1}>m_{2}^{\prime} \tau_{2}\left(m_{2} \tau_{1}<m_{2}^{\prime} \tau_{2}\right)$. This is the desired transition amplitude, which enjoys the following properties.

1. The "orthogonality"

$$
\lim _{\tau_{2} \rightarrow \tau_{1}}<m^{\prime}, \bar{m}^{\prime} ; \tau_{2} \mid m, \bar{m} ; \tau_{1}>=m_{2}^{2} \delta^{2}\left(m^{\prime}-m\right)
$$

2. The divergent-peak on the circle

$$
C_{\infty}:\left(m_{1}^{\prime}-m_{1}\right)^{2}+m_{2}^{\prime 2}+m_{2}^{2}-m_{2} m_{2}^{\prime}\left(\frac{\tau_{2}}{\tau_{1}}+\frac{\tau_{1}}{\tau_{2}}\right)=0
$$

3. The Schrödinger equation in ( the spinor representation of ) the ADM formalism

$$
i \frac{\partial}{\partial \tau_{2}}<m^{\prime}, \overline{m^{\prime}} ; \tau_{2}\left|m, \bar{m} ; \tau_{1}>=\hat{H}\left(m^{\prime}, \overline{m^{\prime}}\right)<m^{\prime}, \overline{m^{\prime}} ; \tau_{2}\right| m, \bar{m} ; \tau_{1}>
$$


The orthogonality (48) is necessary for the amplitude to be consistent with eq.(37).

The circle $C_{\infty}$, where the amplitude becomes divergent, exactly coincides with the classical orbit $C_{\text {c.o. }}$ in eq.(23). Therefore, the amplitude in the quantum theory is distributed around the classical orbit. This result is reasonable from the quantum mechanical viewpoint. This, together with the positive definiteness of eq.(33) and the fact that it is conserved for the solutions of eq.(43), gives a support to the interpretation of the natural inner product (33) as a probability amplitude.

The Schrödinger equation (50) is formally a direct consequence of the fact that eq.(44) solves eq.(42). After lengthy calculations, we see explicitly that the equation

$$
\left(i \tau_{2} \frac{\partial}{\partial \tau_{2}}\right)^{2}<m^{\prime}, \bar{m}^{\prime} ; \tau_{2}\left|m, \bar{m} ; \tau_{1}>=\Delta_{1 / 2}\left(m^{\prime}, \overline{m^{\prime}}\right)<m^{\prime}, \overline{m^{\prime}} ; \tau_{2}\right| m, \bar{m} ; \tau_{1}>
$$

holds. Therefore, the amplitude obtained here can be regarded as that in the spinor representation of the ADM formalism. This follows from the equivalence between the (weight-1/2) spinor representation of the ADM formalism and the canonical quantization of the C-S gravity, which are related via the transformation (39) as is mentioned in the previous section.

\section{The modular invariance}

While we have not considered so far, there is a problem concerning with the modular invariance. Though at first glance it does not appear that our amplitude (47) exhibits the desired transformation property, we will show in this section that this is the case.

Under a modular transformation $\gamma \in \Gamma$ ( $\Gamma$ is the modular group $\left.S L(2, \mathbf{Z}) / \mathbf{Z}_{2}\right)$;

$$
\gamma: m \rightarrow \gamma m \equiv \frac{x m+y}{z m+w}, \quad \text { where } \quad\left(\begin{array}{cc}
x & y \\
z & w
\end{array}\right) \in S L(2, \mathbf{Z})
$$

the wave function $\tilde{\chi}$ should transform as a weight- $\frac{1}{2}$ automorphic form [5], namely spinor:

$$
\gamma: \tilde{\chi}(m, \bar{m}) \rightarrow \tilde{\chi}(\gamma m, \overline{\gamma m})=e^{i \phi_{\gamma}}\left(\frac{z m+w}{z \bar{m}+w}\right)^{1 / 2} \tilde{\chi}(m, \bar{m})
$$

where $\phi_{\gamma}$ is a constant phase factor which represents an abelianization of $\Gamma$. For such $\tilde{\chi}$ 's, the integration region of the inner product (40) can be restricted to the fundamental region:

$$
F=\{m|\Im m>0,| \Re m|\leq 1 / 2,| m \mid \geq 1\} .
$$


Let us construct the modular covariant transition amplitude. First we should be aware that the transformation law of our amplitude can be written as:

$$
\begin{aligned}
\left(\frac{z \bar{m}^{\prime}+w}{z m^{\prime}+w}\right)^{1 / 2} & <\gamma m^{\prime}, \overline{\gamma m^{\prime}} ; \tau_{2} \mid m, \bar{m} ; \tau_{1}> \\
& =\left(\frac{-z m+x}{-z \bar{m}+x}\right)^{1 / 2}<m^{\prime}, \overline{m^{\prime}} ; \tau_{2} \mid \gamma^{-1} m, \overline{\gamma^{-1} m} ; \tau_{1}>
\end{aligned}
$$

If we take the infinite sum

$$
\begin{aligned}
\ll m^{\prime}, \bar{m}^{\prime} ; \tau_{2} \mid m, \bar{m} ; \tau_{1} \gg & \equiv \sum_{\gamma \in \Gamma} e^{-i \phi_{\gamma}}\left(\frac{z \bar{m}^{\prime}+w}{z m^{\prime}+w}\right)^{1 / 2}<\gamma m^{\prime}, \overline{\gamma m^{\prime}} ; \tau_{2} \mid m, \bar{m} ; \tau_{1}> \\
& =\sum_{\gamma \in \Gamma} e^{i \phi_{\gamma}}\left(\frac{z m+w}{z \bar{m}+w}\right)^{1 / 2}<m^{\prime}, \overline{m^{\prime}} ; \tau_{2} \mid \gamma m, \overline{\gamma m} ; \tau_{1}>,
\end{aligned}
$$

the obtained $\ll m^{\prime}, \overline{m^{\prime}} ; \tau_{2} \mid m, \bar{m} ; \tau_{1} \gg$ behaves as a weight-1/2 (or $-1 / 2$ ) automorphic form under the modular transformation of the argument $m^{\prime}$ (or $m$ ). We can therefore regard this infinite sum as a covariant amplitude.

Using this covariant amplitude (53) we can express the evolution of the covariant wave function $\tilde{\chi}$ as:

$$
\tilde{\chi}\left(m, \bar{m} ; \tau_{2}\right)=\iint_{F} \frac{d^{2} m^{\prime}}{\left(m_{2}^{\prime}\right)^{2}} \ll m, \bar{m} ; \tau_{2} \mid m^{\prime}, \overline{m^{\prime}} ; \tau_{1} \gg \tilde{\chi}\left(m^{\prime}, \bar{m}^{\prime} ; \tau_{1}\right) .
$$

This equation can be rewritten as follows. We substitute the definition (53) of the covariant amplitude into (54), and exploit the covariance (51) of $\tilde{\chi}$, the modular invariance of the integration measure, and the mathematical fact [13]:

$$
\bigcup_{\gamma \in \Gamma} \gamma \cdot F=H
$$

where $H$ is the upper-half plane. Then eq.(54) is reexpressed in terms of the original amplitude (47):

$$
\tilde{\chi}\left(m, \bar{m} ; \tau_{2}\right)=\iint_{H} \frac{d^{2} m^{\prime}}{\left(m_{2}^{\prime}\right)^{2}}<m, \bar{m} ; \tau_{2} \mid m^{\prime}, \bar{m}^{\prime} ; \tau_{1}>\tilde{\chi}\left(m^{\prime}, \overline{m^{\prime}} ; \tau_{1}\right) .
$$

We should remark that the covariance of $\tilde{\chi}$ is preserved under the evolution (55) owing to the transformation law (52) of the amplitude. We can thus consider that our amplitude (47) serves as a covariant amplitude when the integration region is extended to the upperhalf plane.

Here we will claim that the physical content is not modified by taking the sum (53). We should notice that for every point $m \in H$ (except fixed points $m=i, e^{i \pi / 3}, e^{2 i \pi / 3}$ ) 
there exists a unique $\gamma \in \Gamma$ such that $\gamma m \in F$ [13]. So we can use proper modular transformations to confine the trajectories or the classical orbits into the fundamental region $F$. These "confined" trajectories or orbits are uniquely determined from the original trajectories or orbits. Morover, two trajectories (or classical orbits) which give the same confined trajectory (or orbit) cannot be distinguished even at the classical stage because two points in the upper-half plane which can transform with each other by an element of the modular group give the same torus. Thus we conclude that taking the infinite sum (53) does not modify any of the physics except that it confines the physical process into the fundamental region $F$.

Since we have obtained the modular covariant amplitude, the issue of the modular invariance is reduced to the problem of searching for the modular covariant wave functions. A set of covariant wave functions can be obtained as follows. We first diagonalize operators $\hat{E} \equiv \tau \hat{H}$ and $\hat{p}_{1}$ in the C-S gravity. Their eigenstates are

$$
\psi^{\left(E, p_{1}\right)}(a, b)=a^{-2 i E-1} \exp \left(i p_{1} \frac{b}{a}\right)
$$

which satisfy

$$
\hat{E} \psi^{\left(E, p_{1}\right)}=E \cdot \psi^{\left(E, p_{1}\right)} \text { and } \hat{p}_{1} \psi^{\left(E, p_{1}\right)}=p_{1} \cdot \psi^{\left(E, p_{1}\right)} .
$$

We sum up these $\psi$ 's with the same $E$ as

$$
\Psi^{(E)}(a, b)=\sum_{p_{1}=\phi_{T}+2 \pi n, n \in \mathbf{Z}} \rho_{E}\left(p_{1}\right) \cdot \psi^{\left(E, p_{1}\right)}
$$

where $\rho$ 's are weights properly chosen so that the $\Psi$ 's are invariant under the modular transformation 3

$$
\gamma:(b, a) \rightarrow(x b+y a, z b+w a) .
$$

Such wavefunctions should be related with the weight-1/2 Maass forms [12] [14] via the integral transformation (39). We will show below that this is indeed the case.

We first perform the inverse transformation (44) of the wavefunction (56) and find

$$
\tilde{\psi}^{\left(E, p_{1}\right)}(m, \bar{m})=N\left(E, p_{1}\right) \tau^{-i E} e^{i p_{1} m_{1}} f_{E, p_{1}}\left(2\left|p_{1}\right| m_{2}\right),
$$

\footnotetext{
${ }^{3}$ Note that $p_{1}$ takes the values which are a constant $\phi_{T}$ plus $2 \pi$ times integrals follows from the requirement that the $\Psi$ 's are invariant up to a constant phase factor under the Dehn twist $T:(a, b) \rightarrow$ $(a, a+b)$.
} 
where $N\left(E, p_{1}\right)$ is an overall constant and $f_{E, p_{1}}$ is given by

$$
\begin{array}{ccc}
f_{E, p_{1}}(z) & = & z e^{-z / 2} \int_{0}^{\infty} d x x^{-2 i E-1}\left(x+\frac{p_{1}}{\left|p_{1}\right|} \frac{1}{x}\right) \exp \left\{-\frac{z}{4}\left(x-\frac{1}{x}\right)^{2}\right\} \\
=e^{-z / 2} z^{i E+1 / 2} & \int_{0}^{\infty} d t & \left(t+\sqrt{t^{2}+z}\right)^{-2 i E}\left\{\left(1-\frac{p_{1}}{\left|p_{1}\right|}\right) \frac{t}{\sqrt{t^{2}+z}}+\left(1+\frac{p_{1}}{\left|p_{1}\right|}\right)\right\} e^{-t^{2}} \\
+e^{-z / 2} z^{-i E+1 / 2} & \int_{0}^{\infty} d t & \left(t+\sqrt{t^{2}+z}\right)^{2 i E}\left\{-\left(1-\frac{p_{1}}{\left|p_{1}\right|}\right) \frac{t}{\sqrt{t^{2}+z}}+\left(1+\frac{p_{1}}{\left|p_{1}\right|}\right)\right\} e^{-t^{2}},(59
\end{array}
$$

which is well-defined in the region $\Re z>0$. We find that this $f_{E, p_{1}}$ is reduced to the following form

$$
\begin{aligned}
& f_{E, p_{1}}(z)=2 \sqrt{\pi}(-i E)^{\frac{\left|p_{1}\right|-p_{1}}{2\left|p_{1}\right|}} W_{\frac{p_{1}}{2 \mid p_{1}}, i E}(z) \\
& \equiv e^{-z / 2}\left[\begin{array}{c}
2^{-2 i E} \Gamma(-i E+1 / 2) z^{i E+1 / 2} F\left(i E+\frac{\left|p_{1}\right|-p_{1}}{2\left|p_{1}\right|}, 1+2 i E, z\right) \\
+\frac{p_{1}}{\left|p_{1}\right|} 2^{2 i E} \Gamma(i E+1 / 2) z^{-i E+1 / 2} F\left(-i E+\frac{\left|p_{1}\right|-p_{1}}{2\left|p_{1}\right|}, 1-2 i E, z\right)
\end{array}\right],
\end{aligned}
$$

where $F(\alpha, \beta, z)$ denotes the confluent hypergeometric function and $W_{\mu, \kappa}(z)$ is the Whittaker function [12]. The proof goes as follows: i) eq.(59) satisfies Whittaker's differential equation

$$
\frac{d^{2}}{d z^{2}} f_{E, p_{1}}(z)=\left[\frac{-E^{2}-1 / 4}{z^{2}}-\frac{p_{1}}{2\left|p_{1}\right| z}+\frac{1}{4}\right] f_{E, p_{1}}(z)
$$

ii) the asymptotic behavior of eq.(59) as $z \rightarrow+\infty$ precisely coincides with that of eq.(60), i.e.

$$
f_{E, p_{1}}(z) \longrightarrow 2 \sqrt{\pi}(-2 i E)^{\frac{\left|p_{1}\right|-p_{1}}{2\left|p_{1}\right|}} e^{-z / 2} z^{\frac{p_{1}}{2\left|p_{1}\right|}} \quad \text { as } \quad z \rightarrow+\infty \quad ;
$$

and iii) the local behavior of eq.(59) in the immediate neighbourhood of $z=0$ is exactly the same as that of eq.(60).

Using the above result, we see that the inverse transform of eq.(57) is expressed as follows:

$$
\begin{aligned}
& \tilde{\Psi}^{(E)}(m, \bar{m})=\tau^{-i E} \sum_{\substack{p_{1}=\phi_{T}+2 \pi n \\
n \in \mathbf{Z}}} \tilde{\rho}_{E}\left(p_{1}\right) \cdot W_{\frac{p_{1}}{2\left|p_{1}\right|}, i E}\left(2\left|p_{1}\right| m_{2}\right) e^{i p_{1} m_{1}}, \\
& \left(\tilde{\rho}_{E}\left(p_{1}\right) \equiv 2 \sqrt{\pi}(-i E)^{\frac{\left|p_{1}\right|-p_{1}}{2\left|p_{1}\right|}} N\left(E, p_{1}\right) \rho_{E}\left(p_{1}\right)\right) .
\end{aligned}
$$

This is the very expression that gives the weight-1/2 Maass forms [12]. We should notice that these covariant wave function $\tilde{\Psi}$ 's satisfy the Schrödinger equation (42) owing to 
Whittaker's equation (61)f and that each term in the sum (63) damps exponentially as $m_{2} \rightarrow \infty$ thanks to eq.(62). The latter property is similar to that of the wavefunctions obtained by Hosoya and Nakao [9], which are weight-0 Maass forms, and so the probability of the occurrence of the singular universes is expected to be extremely small.

In fact, the $\hat{E}$-operator is modular invariant. We do not know whether the physical operators should be modular invariant or not. If that is the case, however, the $\hat{E}$-operator and its eigenfunction $\Psi^{(E)}$ play an important role. Classically, $E$ is connected with the volume $v$ of the time-slice $\Sigma$ ( see eq.(6) ). Thus the state $\Psi^{(E)}$ represents the torus universe with a given "volume" $E$.

Finally we remark that our set of covariant wave functions involves the wave function of the "static universe with a zero-volume" which is obtained by Carlip [5]. Setting $E=0$ and $\phi_{T}=\pi / 6$ in the sum $(63)$ and noting the fact:

$$
W \frac{p_{1}}{2\left|p_{1}\right|}, E=0(z)=\left\{\begin{array}{lll}
e^{-z / 2} z^{1 / 2} & \text { for } & p_{1}>0 \\
0 & \text { for } & p_{1}<0
\end{array}\right.
$$

we have the desired result

$$
\tilde{\Psi}^{(0)}(m, \bar{m})=m_{2}^{1 / 2} \eta^{2}(m),
$$

where $\eta$ denotes the Dedekind $\eta$ function.

\section{Summary and Discussion}

We have computed the transition amplitude in the $2+1$ dimensional Einstein gravity, which describes the evolution of the moduli of a torus $T^{2}$, by way of the C-S gravity. It has a peak on the classical orbit and enables us to interpret the inner product (33) in the C-S gravity as a probability amplitude.

We should notice that this amplitude is obtained via the $C$-S gravity. In principle, we can deduce this result entirely in the ADM's framework, by means of the wave-packet or path integrals. In practice, however, the ADM Hamiltonian (41) is too complicated to

\footnotetext{
${ }^{4}$ Due to the symmetry of the Whittaker function:

$$
W_{\mu, \kappa}=W_{\mu,-\kappa}
$$

we can take $E$ as non-negative provided that the norm of $\tilde{\Psi}$ is well-defined. Though the spectrum of $E$ is continuous for each constituent $\tilde{\psi}$, it is discretized by imposing the covariance on $\tilde{\Psi}$ [12].
} 
carry out the computation. The Schrödinger equation (43) is trivial and we can easily compute the amplitude in the C-S gravity.

Putting our results and Carlip's [5]together, we find an answer to the long-standing problems in the quantum gravity, namely the issue of time and how to interpret the wave function, in the $2+1$ dimentional gravity on a torus. That is, if we use the York-time $\tau \equiv \pi^{a b} g_{a b} \sqrt{g}^{-1}$ as time, and the natural inner product (33) as a probability amplitude, such an amplitude conserves under the evolution in $\tau$, so we can give the probability interpretation to a wave function, which, in turn, describes the quantum evolution of a time-slice $\Sigma$. Besides, the transition amplitude which we have obtained can be regarded as modular covariant in the sense explained in $\S 5$ and thus the issue of the modular invariance is reduced to the problem of constructing the modular covariant wavefunctions. Though we have shown that a set of covariant wave functions are obtained using the weight-1/2 Maass forms, construction of a modular covariant wave function in general requires a considerable understanding of the non-holomorphic modular forms [14] and is left to the future investigation.

We have studied the $2+1$ dimensional quantum gravity in the simplest case, in which the spatial hypersurface has the topology of a torus $T^{2}$. The extension to the case of arbitrary topology is an interesting problem. Since the classical equivalence of the C-S gravity and the ADM formalism has already been proved [15], we expect that this equivalence enables us to compute the transition amplitudes for the case of more complicated topologies. However in the case of a complicated topology ( e.g. a Riemann surface of genus $g \geq 2$ ), it is hard to extract relevant variables which parametrize the phase space. We need a further study to compute such a higher genus amplitude.

Our original motivation to this work was to study the physical meaning of the loop functionals in the $2+1$ dimensional quantum gravity [11]. It turns out that there is a certain difficulty in carrying it out. As is mentioned in $\S 3$, the phase space of the C-S gravity on a torus is composed of three disconnected sectors. Among them, the loop representation, which Ashtekar et.al. constructed [11], is well-defined in the timelike sector. The equivalence of the two formalisms has been established in the spacelike sector alone. Thus the interpretation of the loop functionals in the context of the ADM formalism 
appears to be formidable.尸

In the case of the $3+1$ dimensional gravity, the phase space of the Ashtekar formalism [16] naturally includes that of the ADM formalism because the former contains the singularity of the latter i.e. $\operatorname{det}\left(g_{a b}\right)=0$. The "quantum tunneling" occurring in the Ashtekar formalism, which is confirmed in the minisuperspace model [17], is due to such a situation.

Similarly, in the $2+1$ dimensional case, the phase space of the C-S gravity is in fact larger than that of the ADM formalism. Therefore, if we reconstruct the theory so that we can handle all disconnected sectors, possibly the tunneling can appear from the Euclidean to the Lorentzian spacetime. The physical interpretation of the loop representation will also be given. This interpretation, in turn, may give some useful insights into the $3+1$ dimensional loop representation proposed by Rovelli and Smolin [18], and therefore to the quantum gravity in $3+1$ dimensions.

Acknowledgments

I would like to thank Prof. K. Kikkawa and H. Kunitomo for useful discussions and helpful comments on the manuscript. I am also grateful to Prof. H. Itoyama for his encouragement and a careful reading of the manuscript.

\section{References}

[1] S. Deser, R. Jackiw and G. 't Hooft, Ann. Phys. 152 (1984) 220;

S. Deser and R. Jackiw, Ann. Phys. 153 (1984) 405

[2] E. Martinec, Phys. Rev. D30 (1984) 1198

[3] E. Witten, Nucl. Phys. B311 (1988) 46

\footnotetext{
${ }^{5}$ Recently, Louko and Marolf [19] have proposed the quantum theory which is defined on almost the whole phase space $\mathcal{M}$ of the C-S gravity. Using this, it is possible to elucidate the relation between the $\mathrm{ADM}$ formalism and the loop representation.
} 
[4] S. Carlip, Nucl. Phys. B324 (1989) 106;

J.E. Nelson and T. Regge, Nucl. Phys. B328 (1989) 190

[5] S. Carlip, Phys. Rev. D42 (1990) 2647; D45 (1992) 3584

[6] A. Anderson, preprint Imperial/TP/92-93/02, gr-qc/9210007

[7] E. Witten, Nucl. Phys. B323 (1989) 113;

Y. Fujiwara, S. Higuchi, A. Hosoya, T. Mishima and M. Siino, Phys. Rev. D44 (1991) 1756 and 1763 ;

S. Carlip, preprint UCD- 92-16, hep-th/9206103

[8] V. Moncrief, J. Math. Phys. 30 (1989) 2907

[9] A. Hosoya and K. Nakao, Prog. Theor. Phys. 84 (1990) 739

[10] A. Hosoya and K. Nakao, Class. Quant. Grav. 7 (1990) 163

[11] A. Ashtekar, V. Husain, C. Rovelli, J. Samuel and L. Smolin, Class. Quant. Grav. 6 (1989) L185.

[12] J.D. Fay, J. Reine Angew. Math. 293 (1977) 143.

[13] J. P. Serre, A. Course in Arithmetic ( Springer-Verlag, New-York, Heidelberg, Verlin ) Chap. VII.

[14] Modular Forms, ed. by R.A. Rankin ( Ellis Horwood, Chichester, 1984 ).

[15] G. Mess, preprint IHES/M/90/28;

S. Carlip, Class. Quant. Grav. 8 (1991) 5.

[16] A. Ashtekar, Phys. Rev. D36 (1987) 295;

T. Jacobson and L. Smolin, Nucl. Phys. B299 (1988) 295.

[17] H. Kodama, Phys. Rev. D42 (1990) 2548.

[18] C. Rovelli and L. Smolin, Nucl. Phys. B331 (1990) 80.

[19] J. Louko and D. M. Marolf, preprint SU-GP-93/7-6, gr-qc/9308018. 


\section{Figure Captions}

Fig.1a A torus universe embedded into the Minkowski space.

We take the $T$-axis as a baseline $B$ and draw lines which are obtained by transforming the baseline by $\Lambda_{1}, \Lambda_{2}$ and $\Lambda_{1} \Lambda_{2}$ ( see eq. (17)), The torus universe is expressed by the region inside these lines, with the boundary surface identified by the above transformations.

The case of $(a, u)=(1,0),(b, w)=(0,1)$ is shown explicitly. The torus universe is the shadowed region inside the bold lines, where the two dotted lines and the two dashed lines are respectively identified.

Fig.1b An evolution of the time-slice developed into the $(Y, \theta / \tau)$-plane.

The baseline $\mathrm{B}$ is the $T$-axis, i.e. $Y=\theta=0$. Three parallelograms (drawn by bold lines) intersecting with $\mathrm{B}$ at $T=0,1$ and 2 represent time-slices at the time $\tau=\infty, 1$ and $1 / 2$, respectively. On each parallelogram, opposite sides are regarded to be identified. The figure shows how a "wide" torus shrinks to the wire-like singularity as time elapses.

From this picture, if we wish, we can deduce the trajectory of the moduli parameters, which becomes a semicircle centered on the $m_{1}$-axis ( see ref. [10]). Note that a point $(a, b ; w, u)$ in the $\mathrm{C}-\mathrm{S}$ phase space corresponds to a $\tau$-evolution of the torus universe.

Fig.2 The "classical orbit" $C_{\text {c.o. }}$ of moduli parameters.

$C_{\text {c.o. }}$ is a circle in the upper-half plane. We can see that the orbit expands as $\tau_{2}$ gets larger (or smaller), starting from $\tau_{1}$. 